ERRATA

\title{
A Note on the Kepler Problem in a Space of Constant Curvature.
}

\section{N. Katayama}

Department of Mechanical Engineering, Osaka Prefectural College of Technology Neyagawa, Osaka 572, Japan

(Nuovo Cimento B, 105, $113(1990)$ )

PACS 03.65 - Quantum theory; quantum mechanies.

PACS 99.10 - Errata.

The expressions of Hamiltonian (2) must be

$$
H=\frac{1}{2}\left(1+\frac{1}{4} K r^{2}\right)^{2}\left(\sum_{i=1}^{3} p_{i}^{2}\right)+\left(1-\frac{1}{4} K r^{2}\right) \frac{\beta}{r} .
$$

The expressions of the radial equation (22) must be

$$
\frac{\mathrm{d}^{2}}{\mathrm{~d} y^{2}} R(y)+\frac{2 G^{\prime}(y)}{G(y)} \frac{\mathrm{d}}{\mathrm{d} y} R(y)-2 R(y)(V(y)-E)=\frac{k(k+1)}{G^{2}(y)} R(y) .
$$

Equations (28) and (29) must be

$$
\begin{gathered}
Q_{+} Q_{-} S_{k+1}(\chi)-\left\{\frac{\beta^{2}}{-K(k+1)^{2}}+k(k+2)+\frac{2 E}{-K}\right\} S_{k+1}(\chi)=0, \\
Q_{-} Q_{+} S_{k}(\chi)-\left\{\frac{\beta^{2}}{-K(k+1)^{2}}+k(k+2)+\frac{2 E}{-K}\right\} S_{k}(\chi)=0 .
\end{gathered}
$$

And the introduced inner product (30) can be defined as

$$
\left(S_{k}(\chi), S_{m}(\chi)\right)=(-1 / K) \int_{0}^{\infty} S_{k}^{*}(\chi) S_{m}(\chi)(\sinh (\chi))^{2} \mathrm{~d} \chi .
$$

On the other hand, the inequality (32) should be derived from (28) and (29) as

$$
\frac{\beta^{2}}{-K(k+1)^{2}}+k(k+2)+\frac{2 E}{-K} \geqslant 0 \text {. }
$$

